\title{
Reconstruction of midfacial defects
}

\author{
Bianca Lago-Beack ${ }^{1}$, Julio Acero-Sanz ${ }^{2}$ \\ ${ }^{1}$ Ramón y Cajal and Puerta de Hierro University Hospitals, University of Alcalá, Madrid, Spain; ${ }^{2}$ Head of Department Oral and Maxillofacial \\ Surgery Ramón y Cajal and Puerta de Hierro University Hospitals, University of Alcala, IRYCIS, Madrid, Spain \\ Contributions: (I) Conception and design: J Acero-Sanz; (II) Administrative support: J Acero-Sanz; (III) Provision of study materials or patients: J \\ Acero-Sanz; (IV) Collection and assembly of data: B Lago-Beack; (V) Data analysis and interpretation: Both authors; (VI) Manuscript writing: Both \\ authors; (VII) Final approval of manuscript: Both authors. \\ Correspondence to: Dr. Bianca Lago-Beack, MD, FEBOMS. Servicio de Cirugía Oral y Maxilofacial, Hospital Universitario Ramón y Cajal, 6 a Planta - \\ Centro, Carretera de Colmenar Viejo, km 9,100 CP 38034, Madrid, Spain. Email: bianchilago@gmail.com; bianchasoonhee.lago@salud.madrid.org.
}

\begin{abstract}
Defects of the maxilla and midfacial structures following trauma, benignant or malignant tumor ablation are a challenge for the reconstructive surgeon because of the importance of physical appearance and speech, swallowing and visual functions in which this anatomical area is involved. The bony and soft tissues constituting the midface provide much of the facial contour and profile giving each person its personal appearance. The aim of this paper is to review the methods of reconstruction after oncological resection of the middle third of the face and to present our reconstructive protocol. Reconstructive methods available to restore the defect after oncologic resection in this area can include: (I) obturators; (II) local or regional pedicled flaps; (III) microvascular flaps (forearm, rectus abdominis, fibula, iliac crest, scapula flap, etc.). Repair of a midfacial defect should be based on the size, location and type of tissues involved. It is important to understand the complex three-dimensional anatomy of the maxilla so as the interrelation to adjacent structures of the midface prior to approach the decision-making plan. Indications and disadvantages of the different techniques are reviewed. New technological developments including virtual planning, 3D printing and navigation can contribute to increase safety and accuracy in oncologic resection and reconstruction of the midface.
\end{abstract}

Keywords: Midface; reconstruction; free flaps; computer aided design

Received: 24 May 2021; Accepted: 04 June 2021; Published: 10 September 2021.

doi: 10.21037/fomm-2021-10

View this article at: https://dx.doi.org/10.21037/fomm-2021-10

\section{Introduction: surgical anatomy}

Defects of the maxilla and midfacial structures following trauma or oncological ablation are a challenge for the reconstructive surgeon because of the importance of physical appearance and speech, deglutition and visual functions in which this anatomical area is involved (1-3).

The concept of the maxilla as a box compound by six walls has been described by many authors $(1,4)$. The roof of the box is the floor of the orbit, giving support to the orbital structures being the most important the ocular globe; the base of this box is the alveolar ridge and the palate. The medial wall is the lateral aspect of the nose and nasolacrimal duct and the anterior wall corresponds to the skin and the underlying soft tissues. The superior wall (orbital floor), the inferior (palatal) and anterior walls (mimic musculature, skin and soft tissues) must be reestablished in order to retrieve the function and the aesthetics lost after tumor ablation. The importance lies in the function that these anatomical landmarks have:

* Bone replacement must be addressed to maintain position of the orbital globe.

* Maintenance of facial contour and projection.

* Bone stock for osseointegrated implants and posterior dental prosthesis rehabilitation.

* Closure of palatal defects to avoid oronasal and oroantral symptoms and ensure correct speech and air flow within the nose. 


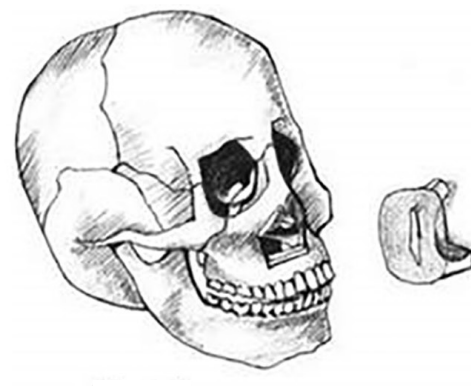

Type 1

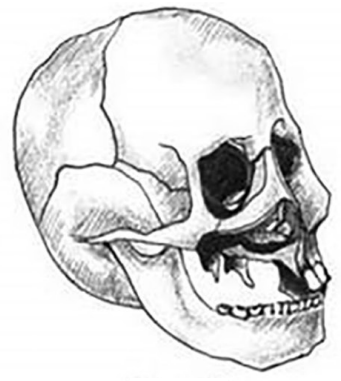

Type 2
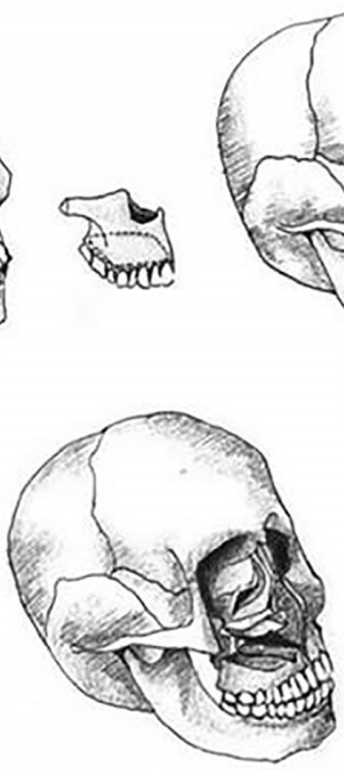

Type 4

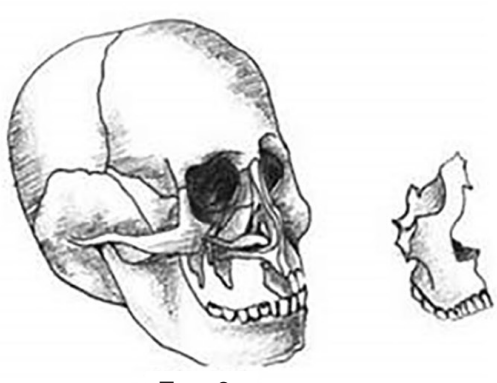

Type 3a

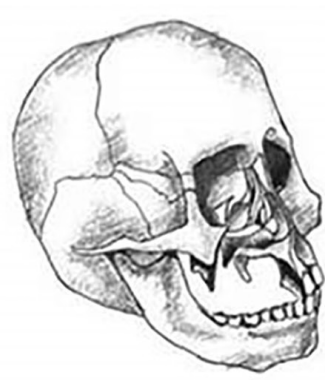

Type 3b

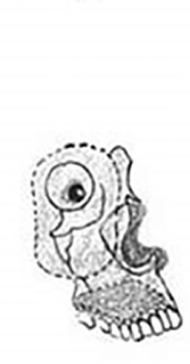

Figure 1 Classification system for maxillectomy and midfacial defects with corresponding tissue defect according to Cordeiro and Santamaria (1).

Repair of a midfacial defect should be based on the size, location and type of tissues involved. It is important to understand the complex three-dimensional anatomy of the maxilla so as the interrelation to adjacent structures of the midface prior to approach the decision-making plan.

\section{Classification}

There is not a systematic approach established when reconstructing complex maxilla or midfacial defects. Many classifications have been described during the last years to guide the reconstruction of the midface after surgical ablation, taking into account diverse aspects like prosthodontic, anatomical and surgical techniques. Many authors aimed to classify midface defects but there is no consensus yet on which one is the best to use.

Initially, prosthetic-based approaches were the standard. Defects were based on anatomical characteristics without focusing on reconstruction options. The continuous evolution of surgical-based reconstructions with the introduction of microvascular surgery had set a paradigm shift that forced to include new parameters in the continuous changing field of midface reconstruction. Better understanding of flap anatomy, inset and geometry, and the increased survival rate of free flaps contributed to the fact that this reconstruction option is considered nowadays as the technique of choice for repairing midface defects $(5,6)$.

Quality of life (QoL) questionnaires and comparative studies comparing different reconstructive options show evidence of better psychosocial and functional outcomes in patients that undergo free flap surgery than those with prosthesis-based rehabilitation (7).

Recent literature has become more focused on individualized techniques for specific defects. Most of the papers focus on bonny reconstruction with no emphasis on volumetric issues or specific structures that need to be replaced. The following classification approaches group midfacial defects according to tissue loss and potential rehabilitative options. Each one has its own advantages and disadvantages, with some being less complete and others being complete but not offering specific guidance in terms of reconstructive technique (7).

Cordeiro and Santamaria classified maxillary defects in four groups depending on the lost maxillary borders [type I, limited maxillectomy; type II, subtotal maxillectomy preserving orbital floor; type III, total maxillectomy including six walls, (IIIa) orbital contents preserved, (IIIb) orbital contents exenterated and type IV, orbitomaxillectomy which includes orbital contents but preserves the palate] (1) (Figure 1). 

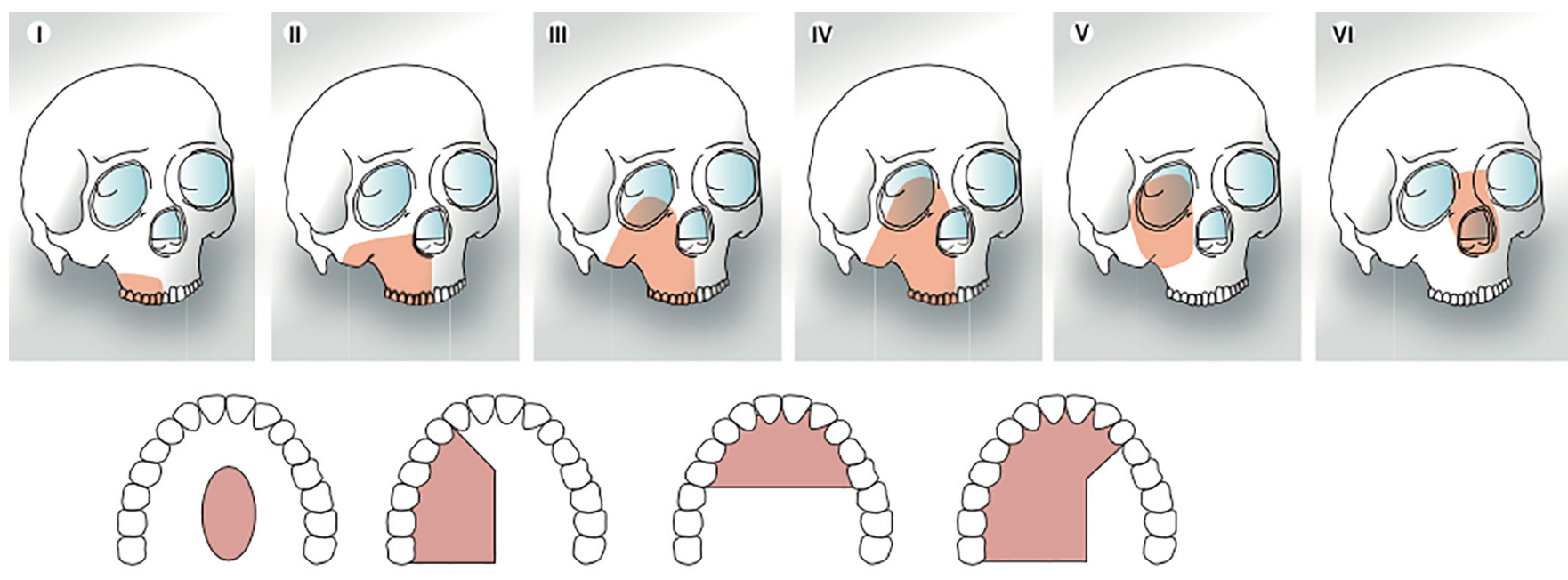

a

b

c

d

Figure 2 Modified classification scheme according to Brown et al. Reconstruction of the maxilla and midface (6).

Another common classification used, is the one described by Brown and Shaw, which defines the defect by its vertical and horizontal extensions. This system aims to guide the reconstruction focusing on the restoration of facial symmetry and projection (vertical component) and proper oral function (horizontal component). Vertical defects are described in classes I-IV and included from isolated palatal defects to orbital exenteration, whilst horizontal components $(\mathrm{a}, \mathrm{b}$ or $\mathrm{c})$ focus on the defects of palate and alveolar ridge involvement. Class V, orbitomaxillary and VI, nasomaxillary defects have been added in the last modification of this classification, and classify ablative defects where palate or maxillary alveolus are not involved $(1,6,8)$ (Figure 2).

This classification system comprehensively includes the potential defects that can affect the different aspects of the midface and its widely used worldwide.

Okay et al. (9) described a different classification of midface defects focusing on the involvement of alveolar ridge and palatal component and the associated functional limitation.

This classification can be useful as a basis for dental prosthetic rehabilitation in maxillary reconstruction and focus on the involvement of tooth-bearing areas of the maxilla, affectation of the premaxilla or a defect extending posterior to the canine (8). Okay's classification also aims to classify the vertical component (class $f$ or $\mathrm{z}$ )—analyzing the involvement of the orbital rim or zygoma (1,9) (Figure 3).

Yamamoto et al. designed a reconstructive algorithm based on three classification scenarios which advocates the use of free flaps predominantly for the reconstruction of maxillary buttresses (10).

\section{Goals of reconstruction}

It is essential for the head and neck surgeon when choosing a certain treatment option to assess the main aspects of midface rehabilitation. These goals include (1,3,6,8,11-17):

* Healed wound.

* Separation of the oral and nasal cavities, restoration of palatal competence.

* Support of the orbital contents and obliteration of the orbital cavity if exenteration is performed.

* Skull base repair.

* Recreation of functional dentition and restoration of facial contours by means of buttresses restoration and facial projection.

The achievement of these goals will depend on the reconstructive method chosen: prosthesis or obturator, locoregional flaps or free flaps.

\section{The reconstructive ladder}

As mentioned before, options for the reconstruction of midface defects include the use of prosthetic obturators, pedicled flaps and free flaps sometimes combined with grafts or alloplastic implants. Although the use of pedicle flaps has decreased lately because of limited arch of rotation, volume and possibility of osseus reconstruction; obturators remain a good solution for selected patients 


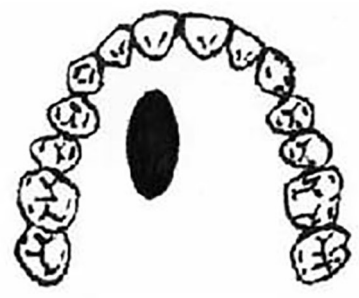

Class 1a

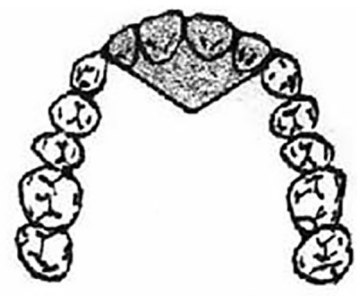

Class $1 b$

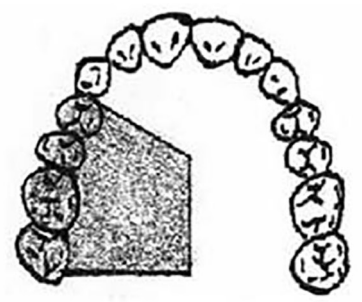

Class $1 b$

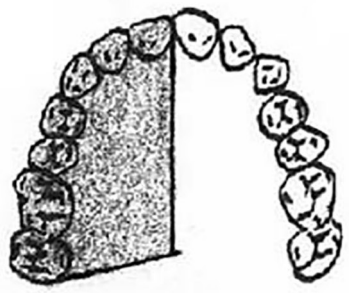

Class 2

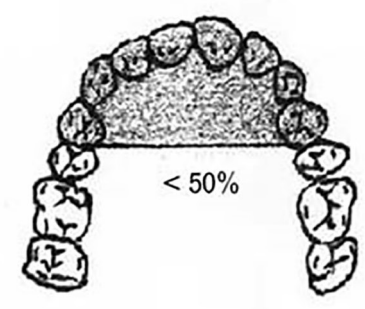

Class 2

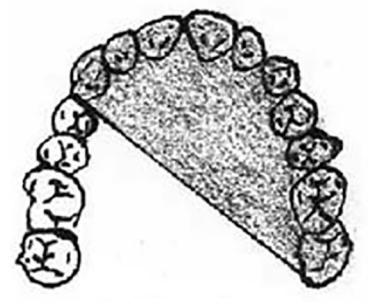

Class 3

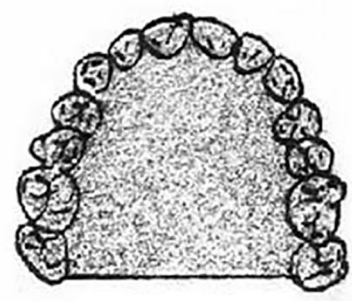

Class 3

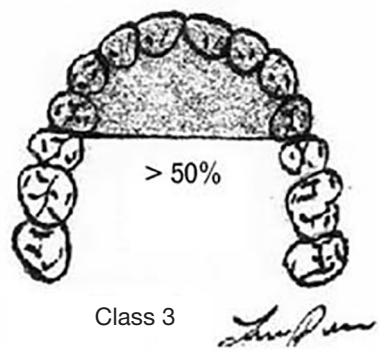

Figure 3 Maxillectomy classification scheme according to Okay et al.

with limited palatal defects or comorbidities among other factors (18). However, for extensive defects including the skull base, orbital floor, orbital contents or soft tissues of the face, obturators are not appropriate being microvascular surgery an excellent alternative for midface reconstruction.

With the incorporation of free flaps reconstruction becomes a feasible single stage procedure without limitations on tissue volume and orientation, vessel geometry and vascular pedicled length (19).

Many donor sites have been described in the literature including radial forearm, rectus abdominis, fibula, scapula, iliac crest and the anterolateral thigh system of free flaps (3). The election of the flap depends on the individual characteristics of the patient and the type of defect (volume of soft tissue, remnant bone and presence of dentition, donor site morbidity) that will guide the surgeon to choose the best reconstructive option for each individual patient (Tables 1 and 2).

\section{Prosthetic obturators}

Obturators can be a simple and functional solution when small defects of the maxilla need to be restored. Limited dimensions and lack of support in the surrounding tissues after tumor ablation constitute their main limitations. Oral function, chewing and swallowing, are preserved with these devices but free flaps have shown to be more efficient on these functions than obturators (20). Separation of both sino-nasal and oral cavities are addressed by obturators which reduces the nasal speech and food and liquid leakage.

Indications can be extended to different type of defects such as small palatal defects not involving the alveolar ridge and palatomaxillary defects posterior to the canine tooth. In our experience use of obturators are limited to (I) secondary reconstruction after free flap or pedicled flap failure; (II) depending on patient's comorbidities or prognosis factors; (III) possibility of recurrence that can preclude any type of autogenous reconstruction (21). 
Table 1 Reconstruction options depending on the extend of the defect

\begin{tabular}{lll}
\hline Small/isolated palatal defects & Extensive palatomaxillary defects & $\begin{array}{l}\text { Extensive maxillary defects not involving } \\
\text { the palate }\end{array}$ \\
\hline $\begin{array}{l}\text { Local flaps (buccal fat pad, } \\
\text { palatal, FAMM) }\end{array}$ & $\begin{array}{l}\text { Locoregional pedicled flap (temporalis muscle or } \\
\text { temporoparietal fascia flaps, submental) } \\
\text { Prosthetic obturator }\end{array}$ & Soft tissue free flap \\
Radial forearm free flap & Bony free flap & Soft tissue flap + bone grafts or alloplasts \\
& Prosthetic obturator if autogenous reconstruction is not feasible \\
\hline
\end{tabular}

FAMM, facial artery miomucosal flap.

Table 2 Reconstruction options according to Brown's classification

Prosthetic obturator/local flaps/ Vascularized free flaps temporalis muscle flap

Class I-Ila, b (Brown)

Class IIc/III-VI (Brown)

\section{Local and vascularized free flaps}

Tissue transfer has become the gold standard in midface reconstruction. The reports show better outcomes with surgical reconstruction when comparing with prosthesis in terms of function and esthetics, especially when large defects are involved. Selection of a specific flap either pedicled or as free tissue transfer depends on factors related to the recipient site such as the size of the defect and characteristics of tissues involved and factors related to the flap such as: length of the vascular pedicled; thickness of the skin, muscle and subcutaneous fat; the volume of the tissue available; the durability and thickness of the bone and the morbidity of the donor site. Microvascular anastomosis in reconstruction of the midface could require long pedicle flaps if the recipient vessels are located in the neck region $(22,23)$.

Local flaps such as the buccal fat pad and palatal, facial artery miomucosal flap (FAMM) can be an option to repair minor postero-lateral defects of the upper maxilla. The temporalis pedicled flap can be indicated in palatal defects or posteroinferior maxillectomy, also in Brown's class IIb, IId defects. If there is an oroantral or oronasal fistulae, the oral cavity should be sealed. The use of the pedicled temporalis muscle flap is a good option to provide adequate and permanent coverage of the defect (Figure 4).

Radial forearm free flap (RFF) is an alternative to restore continuity in deep central palatal defects (Brown's class I and IIa,b) as it can provide dual skin lining to both the nasal floor and the palate surfaces because of its great pliability and with minimum bulk.

Fibula free flap (FFF) is indicated in Brown's class IId, III, IV, V defects. This flap is often used in type II maxillary defects. It is a reliable and feasible flap. Moreover, it offers the possibility to complete a final restoration with dental implants. The pedicle has a favorable length, the bone stock ranges from 20 to $25 \mathrm{~cm}$ and allows for a two-team approach. It gives support to the nose and orbit avoiding collapse of the midfacial soft tissues. The pliable skin paddle can be used for either intraoral or cutaneous reconstruction. Implant supported dental prosthesis can be placed, so masticatory function and lip support are reestablished (6). This flap shows to be extremely versatile for restoring not only the alveolar ridge and framework of the midface but also giving support to the orbital contents by reproducing the orbital rim and allowing the settlement of titanium mesh when the orbital floor needs to be removed.

Orientation of the skin paddle and the requirement of osteotomies with deep angles between the bone fragments can be very challenging when using this flap. The size of the skin paddle might be a limitation when extended resections of palatal mucosa and skin are performed and need to be restore with the flap skin island. Thus, according to some authors, this flap might not represent an adequate reconstruction option for class III and IV defects (Figure 5).

Iliac crest free flap (ICFF) can be used in Brown's class II, IIIb, c, d, IV defects. Based on the deep circumflex iliac artery (DCIA) vascular pedicle. This flap has enormous advantages when used for midfacial reconstruction. Specifically, it offers a great amount of bone that could be remodeled to reconstruct the orbital rim and restore facial harmony. It provides enough bone for implant placement and dental rehabilitation. The amount of oblique interne muscle varies depending on the dead space and the resulting 

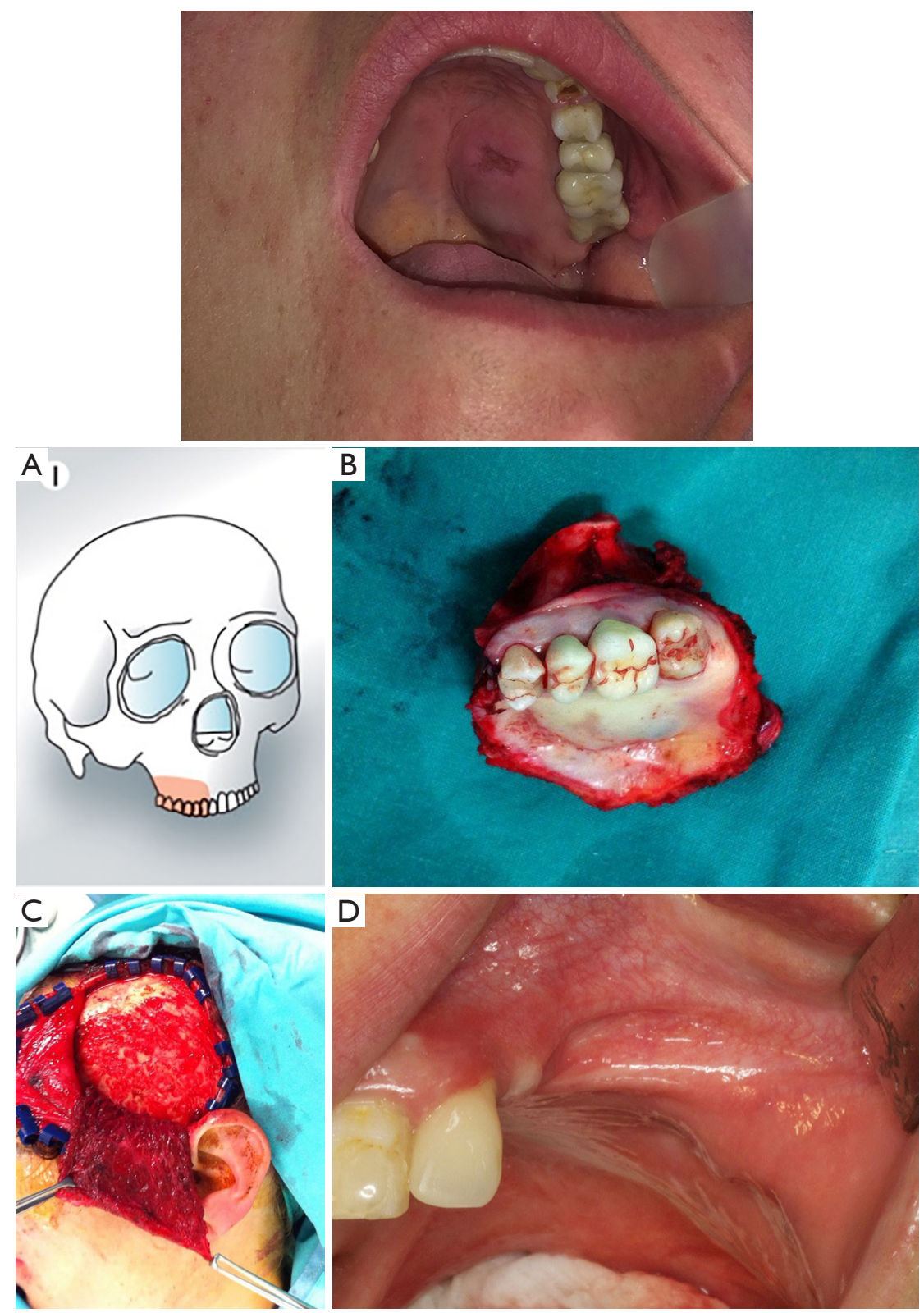

Figure 4 Adenocarcinoma of the maxilla in a 49-year-old female patient. (A) Brown's classification of the defect; (B) surgical specimen; (C) raising of temporalis muscle pedicle flap; (D) postoperative results after 6 months of follow up.

oral fistulae and palatal defect after tumor removal. The orbital contents if exenterated must be taken into account when addressing the volume of the muscle harvested. The only disadvantage found when using the ICFF is the limited length of the vascular pedicled that can difficult the inset of the flap (Figure 6).

Scapula osteocutaneous flap (SOCFF): flaps based on the subscapular artery system offer great advantages for complex midfacial reconstruction. Mostly useful in Brown's class III-IV defects, the soft tissue component of this flap can be rotated around the bone stock with great freedom. It is particularly helpful in defects involving both orbital floor/zygoma and palate. The inclusion of the angular branch of the toracodorsal vessel allows the possibility to harvest the tip and the lateral border of the scapula simultaneously $(1,24,25)$. This flap is extremely useful for 

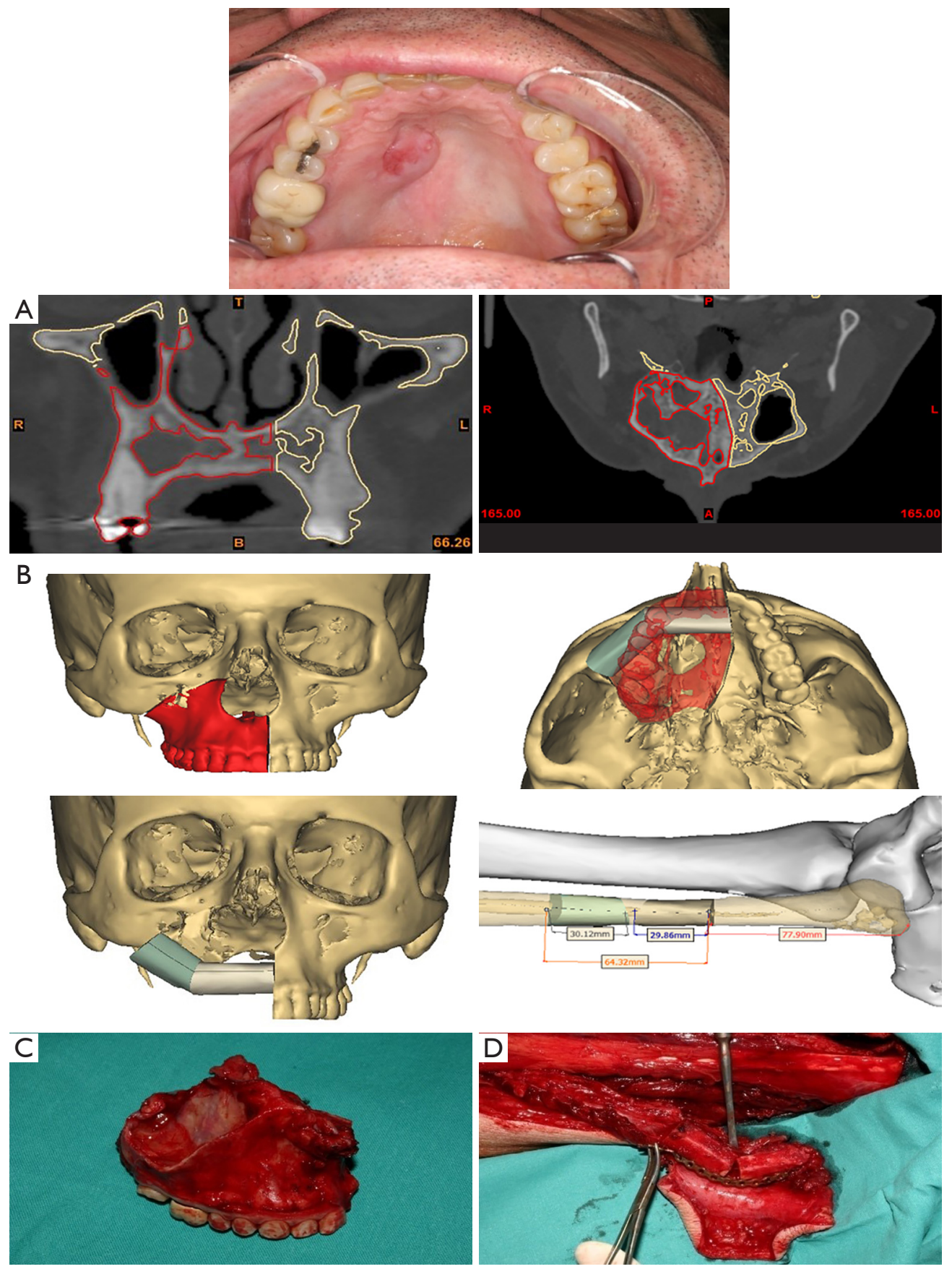

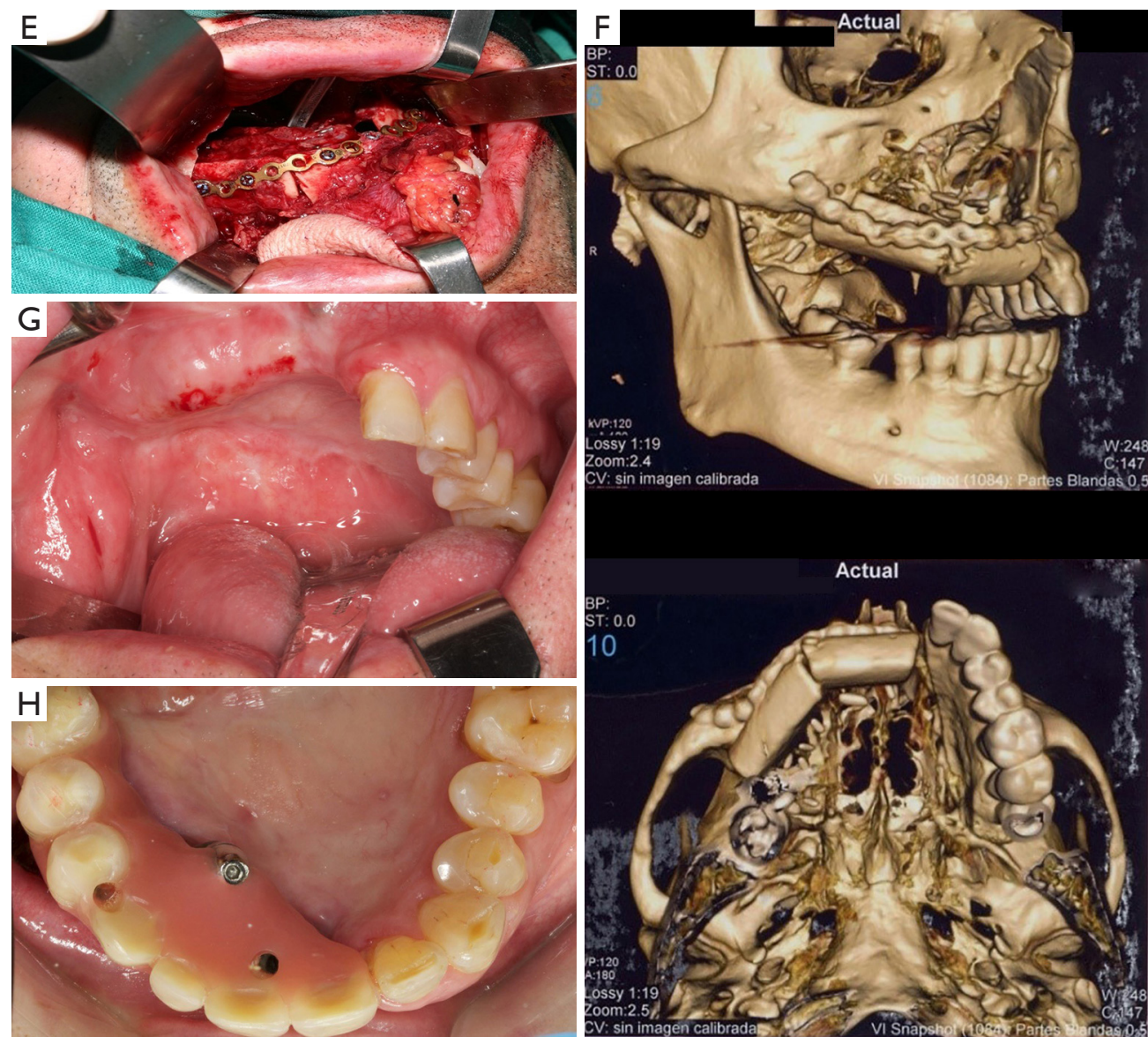

Figure 5 Adenoid cystic carcinoma of the maxilla in a 53-year-old male patient. (A) Superimposition of the CT scan showing the osseus structures and the tumor (planned resection in red); (B) 3D virtual surgical planning of the resection and fibula flap reconstruction; (C) surgical specimen; (D) surgical cutting guides to rise an osteo-cutaneous fibula free flap; (E) insetting of the flap and fixation with titanium plates and screws; (F) postoperative CT control scan; (G) intraoral postoperative result; $(\mathrm{H})$ final result with dental implant supported prosthesis.

reconstructing total maxillectomy defects associated with orbital exenteration. In this sense, the bone can be used for replacing the palate and the infraorbital area, the muscular component (latissimus dorsi or anterior serratus) is usually used for oral reconstruction and the skin component allows the reconstruction of large defects of the face, cheek and palate. The main disadvantages are the difficulty to place osseointegrated implants por dental restoration for its little bone thickness and the difficulty of working with two teams at the same time being more time consuming. Furthermore, the length of the pedicle can be short, and the bone can be hard to orientate in the three-dimensional aspect of the defect (Figure 7).

Soft tissue free flaps like the anterolateral thigh flap, the latissimus dorsi flap and the rectus abdominus provide adequate bulk and volume, seal dead spaces within the defect and have many characteristics as versatility, reshaping of facial contour and palatal closure. However, the reconstruction of the bone structures is not possible by using these flaps (13). They are indicated especially in Brown's class $\mathrm{V}$ defects, were the palate and alveolar ridge remain intact and bone is generally not required (1). The main objectives when using soft tissue flaps in midface defects of these characteristics consist in separating the cranial base of the sinonasal tract and facial structures, support the repaired dura and prevent cerebrospinal fluid leakage, obliterate the orbital defect and provide sufficient depth for an orbital prosthesis. Furthermore, the mentioned flaps offer a long pedicle highly suitable for skull base and midfacial reconstruction (Figure 8). 


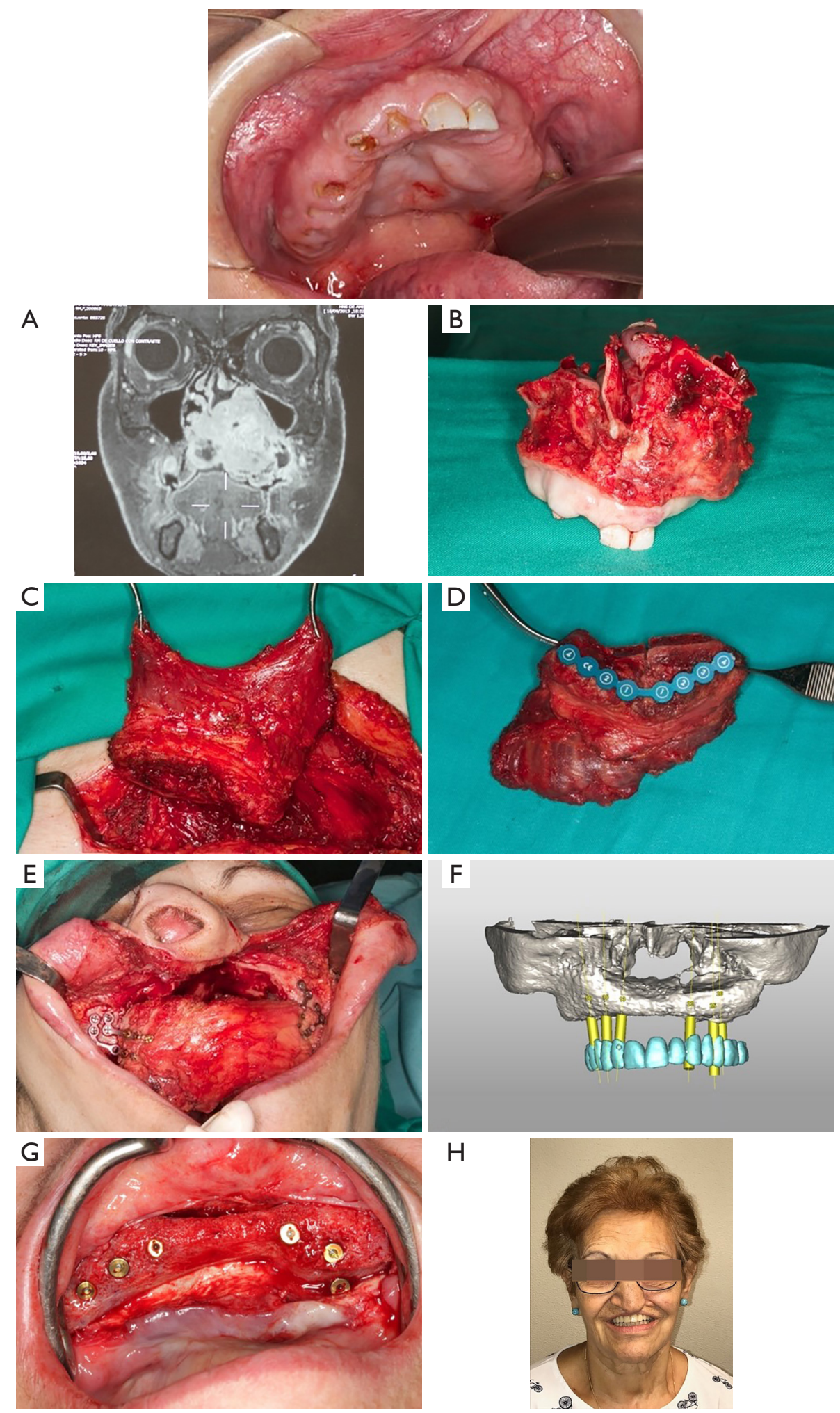

Figure 6 Adenoid cystic carcinoma of the maxilla in a 72-year-old female patient. intraoral examination. (A) MRI; (B) surgical specimen; (C) iliac crest free flap including the internal oblique muscle; (D) modeling of the bone flap. Osteotomy of the iliac crest bone; (E) insetting of the flap after tumor ablation; (F) dental implant 3D planning; (G) dental implant placement; (H) final facial aspect. 

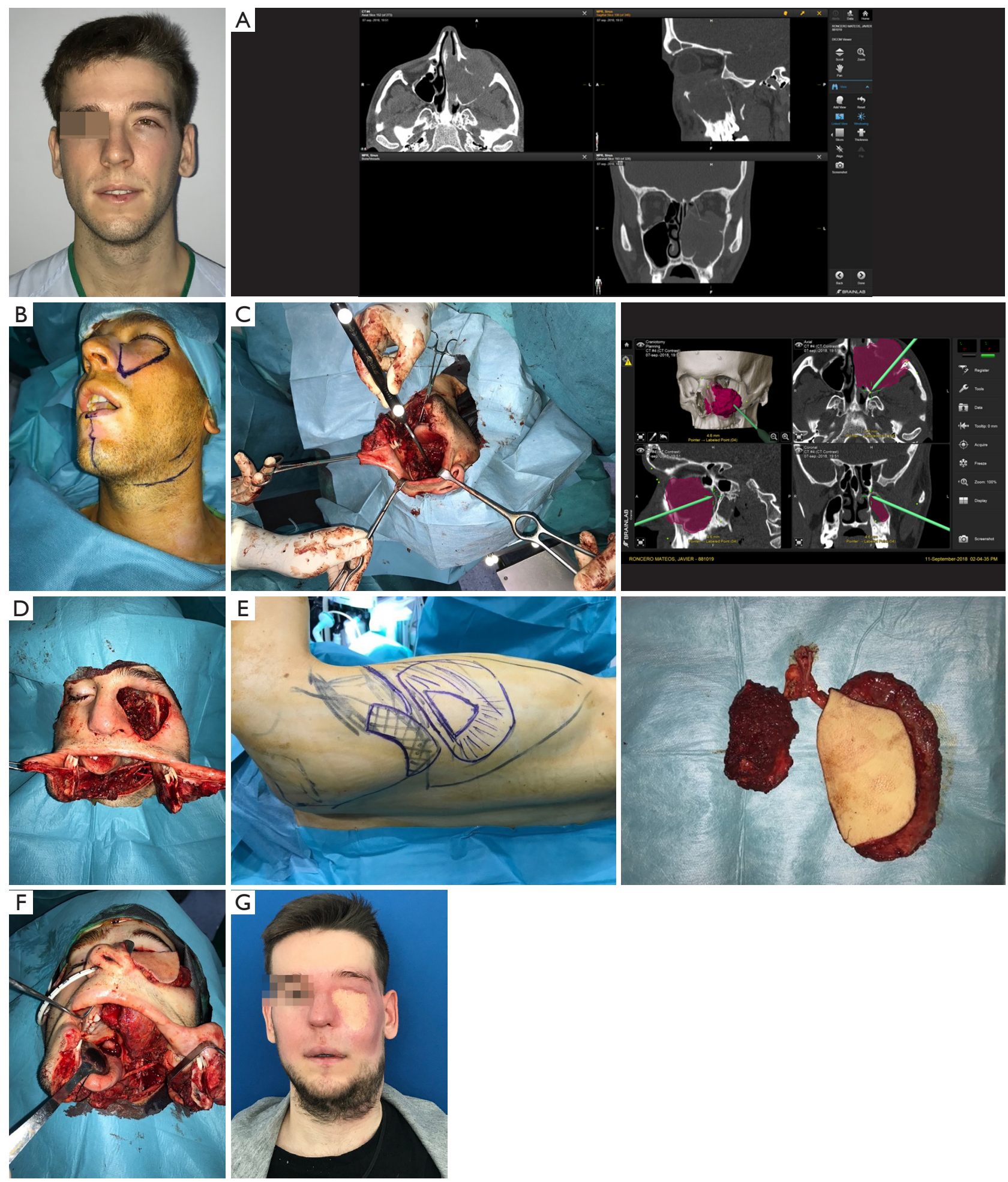

Figure 7 Rabdomiosarcoma involving the orbitomaxillary region in a 23-year-old male patient. (A) CT showing the lesion; (B) combined transmandibular/transfacial approach; (C) intraoperative navigation of the tumor resection; (D) orbitomaxillary defect after tumor ablation; (E) dorso-scapular free flap. Chimeric flap including the latissimus dorsi and the tip of the scapula; (F) insetting of the flap and vascular anastomosis; $(G)$ postoperative result after 3-month of follow-up. 

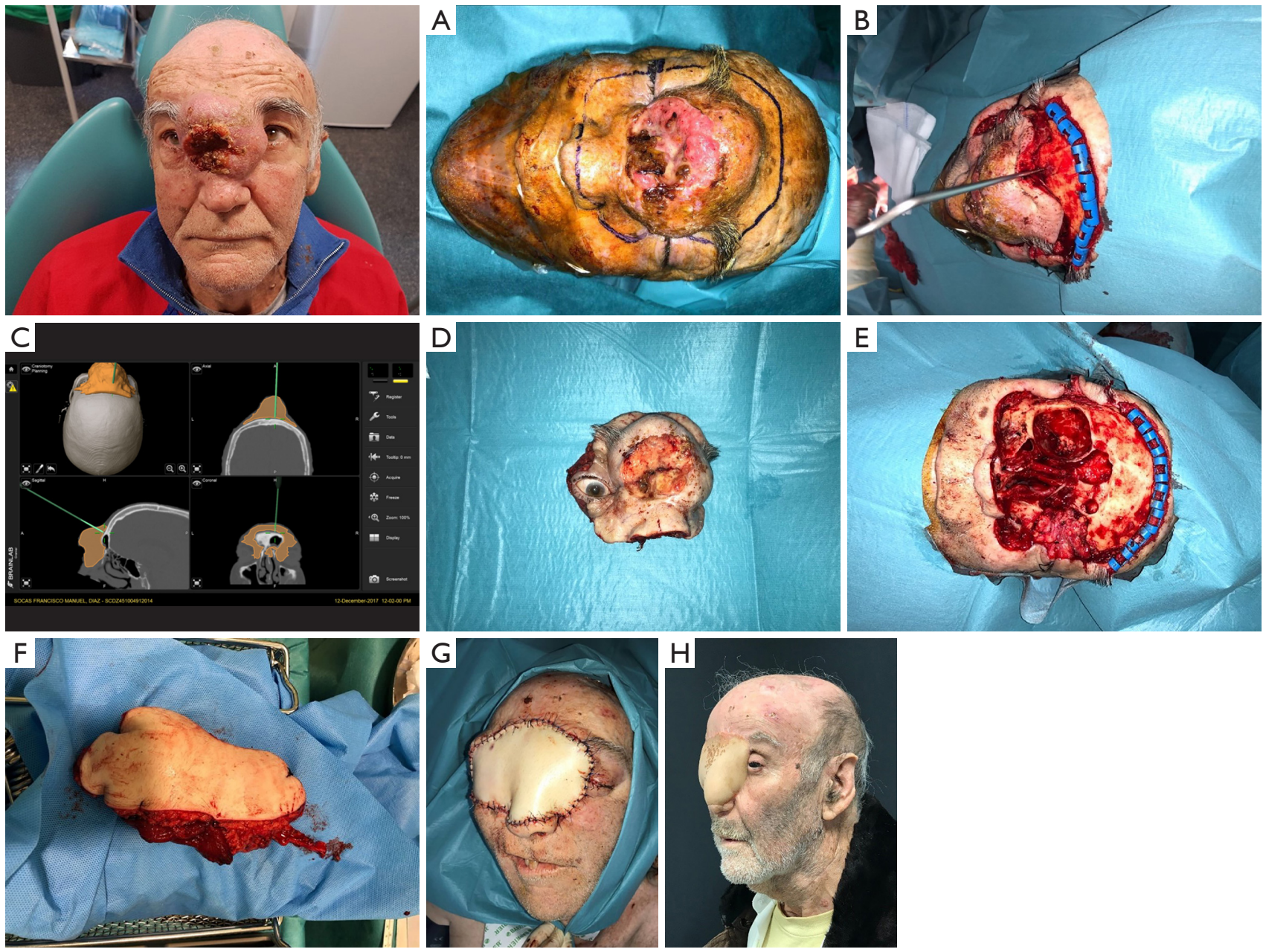

Figure 8 Cutaneous epidermoid carcinoma affecting the naso-orbito-ethmoidal region in a 73-year-old male patient. (A) Surgical resection design; (B) bicoronal approach; (C) intraoperative navigation of the surgical margins; (D) surgical specimen comprising the right orbit and centrofacial region; (E) midface defect; (F) reconstruction with a latissimus dorsi flap; (G) final result after insetting of the microsurgical flap; (H) late postoperative result.

\section{Virtual surgical planning and computer-aided- design/computer-aided-modeling (CAD/CAM) technology}

Over the past years, the evolution of microsurgical techniques and its implementation in reconstructive surgery, especially in the midface that is the main issue in this paper, has been favoured by advances in computer software and 3D manufacturing. Occasionally, the defects turn to be technically difficult to reconstruct so are the inset of the flaps especially those with bony segments, so the implementation of virtual surgery and manufactured specific guides facilitate the execution of the surgery and achieve more accurate results. Virtual reconstruction may be performed, margins of resection, design and setting of flaps can be planned prior to surgery and surgical guides may be manufactured with $3 \mathrm{D}$ printing machines or virtually thus it will help during the procedure and will shorten the surgical time.

Virtual surgical planning is becoming a standardized tool in the field of reconstructive surgery due to its many benefits which include increased accuracy, improved operative efficiency and enhanced outcomes. This technology is useful in craniofacial reconstruction that ranges from trauma to oncological defects among others $(7,11,26,27)$.

Surgical accuracy is required to restore facial symmetry, appearance and function; however, this task is complicated because of the complexity of this anatomical construct that 
encompasses the maxilla and other midfacial structures like the orbit, palate and paranasal sinuses.

$\mathrm{CAD}$ and CAM have been refined in the past years to be an easily and useful technology for preoperative planning and execution of surgery (11), and its implementation includes the following benefits: (I) shortens the length of operating time, (II) reduces flap ischemia time, (III) increases the bone-to-bone contact between osseous flaps and the remnant bone, (IV) improves dental alignment and aesthetic contour, $(\mathrm{V})$ reduces complication rates and (VI) increases reconstruction accuracy, in comparison to traditional oncologic and reconstructive surgery whose final outcomes relied mostly on the surgeon's experience. Virtual surgical planning and CAD/CAM technology requires a multi-stage approach that can enlarge the process globally, so timing must be consider when the treatment plan is being designed.

Computer-assisted surgery is based on four specific phases that must be accomplished in order to achieve predictable and planned outcomes: (I) planning, (II) modeling, (III) surgery, (IV) evaluation.

(I) Planning: a high resolution CT scan with thin cuts and $3 \mathrm{D}$ reconstruction is made of the craniofacial skeleton and the donor site, e.g., lower limb-fibula free flap. The images are forwarded to the modeling company.

A videoconference then, is made between the surgical team and the bioengineers. Resection and reconstruction are virtually planned, taking into account factors such as resection margins, osteotomies performed in the donor bone and placement of the vascularized free flap in the surgical defect.

(II) Model manufacture: $3 \mathrm{D}$ printed models are manufactured of the craniomaxillofacial area of interest along with specific cutting guides for both the tumor resection and the donor site where the flap is going to be raised for reconstruction. It also allows for manufacturing individualized reconstruction plates or plate bending templates depending on the surgeon's preferences.

(III) Surgery: the models, cutting guides and implants previously described are used during surgery. In this phase, osteotomies in the maxilla or mandible are made based on the resection guides for tumor ablation and then the osseous flap is harvested, cut and osteotomies are made in situ with the cutting guides attached and the reconstruction plate is fixed before devascularization of the flap.

(IV) Evaluation: control after surgery must be made by means of clinical examination and postoperative CT scan in order to check if goals of treatment planning have been accomplished. Restoration of function and aesthetics are evaluated and QoL questionnaires are given to the patient (Figure $5 A, B, F)$.

In difficult cases, intraoperative navigation helps in the implementation of procedure plans at the site of surgery. Intraoperative 3D imaging allows an intraoperative final control to be made and the outcome of the surgery to be validated (28). In terms of midfacial reconstruction, intraoperative navigation allows assessing surgical margins in midfacial resection within the orbit (floor, medial wall and posterior margin), cranial base structures or the palatal aspect of the resection among others. It also controls orbital volume and globe position to prevent orbital asymmetry that can lead to hypoglobus and enophthalmos, diplopia and when it affects the lower eyelid, ectropion, epiphora, globe exposure and visual complications (29).

Furthermore, it helps to avoid malpositioning of bone fragments and inserted implants when soft tissue or bony grafts have to be used for reconstruction purposes (Figures $7 A, C, 8 B, C)$.

\section{Conclusions}

Midface reconstruction is a challenging field because of the importance of this anatomical region functionally and aesthetically in the patient's life and health. Threedimensional comprehension of the midface must be considered among other factors in order to assess the treatment goals planned before selecting the best surgical procedure. The advantages of microvascular surgery involve a single stage procedure, most of the time with a two-team approach, that allows restoring bone and soft tissue loss which seals the oral cavity, rebuilds the midfacial skeleton providing support for the overlying tissues, allows for dental rehabilitation and reestablishes facial contour maintaining patient's profile and QoL.

Over the past years, the technological innovations such as computer-assisted surgery have contributed to improve safety and accuracy of midfacial reconstruction. Virtual planning of resection and later reconstruction, computerassisted design and manufacture (CAD-CAM) surgical guides and navigation-assisted surgery are showing to be useful techniques in reconstruction. The new digital technologies will contribute to improve results in this field.

\section{Acknowledgments}

Funding: None. 


\section{Footnote}

Provenance and Peer Review: This article was commissioned by the Guest Editor (Paolo Cariati) for the series "Microvascular reconstruction of head and neck oncological defects-state of the art" published in Frontiers of Oral and Maxillofacial Medicine. The article has undergone external peer review.

Conflicts of Interest: Both authors have completed the ICMJE uniform disclosure form (available at https:// fomm.amegroups.com/article/view/10.21037/fomm-2021$10 /$ coif). The series "Microvascular reconstruction of head and neck oncological defects-state of the art" was commissioned by the editorial office without any funding or sponsorship. The authors have no other conflicts of interest to declare.

Ethical Statement: The authors are accountable for all aspects of the work in ensuring that questions related to the accuracy or integrity of any part of the work are appropriately investigated and resolved.

Open Access Statement: This is an Open Access article distributed in accordance with the Creative Commons Attribution-NonCommercial-NoDerivs 4.0 International License (CC BY-NC-ND 4.0), which permits the noncommercial replication and distribution of the article with the strict proviso that no changes or edits are made and the original work is properly cited (including links to both the formal publication through the relevant DOI and the license). See: https://creativecommons.org/licenses/by-nc-nd/4.0/.

\section{References}

1. Cordeiro PG, Santamaria E. A classification system and algorithm for reconstruction of maxillectomy and midfacial defects. Plast Reconstr Surg 2000;105:2331-46; discussion 2347-8.

2. Dalgorf D, Higgins K. Reconstruction of the midface and maxilla. Curr Opin Otolaryngol Head Neck Surg 2008;16:303-11.

3. Futran ND, Mendez E. Developments in reconstruction of midface and maxilla. Lancet Oncol 2006;7:249-58.

4. McCarthy CM, Cordeiro PG. Microvascular reconstruction of oncologic defects of the midface. Plast Reconstr Surg 2010;126:1947-59.

5. Alam D, Ali Y, Klem C, et al. The Evolution of Complex
Microsurgical Midface Reconstruction: A Classification Scheme and Reconstructive Algorithm. Facial Plast Surg Clin North Am 2016;24:593-603.

6. Brown JS, Shaw RJ. Reconstruction of the maxilla and midface: introducing a new classification. Lancet Oncol 2010;11:1001-8.

7. Chang EI, Hanasono MM. State-of-the-art reconstruction of midface and facial deformities. J Surg Oncol 2016;113:962-70.

8. Badhey AK, Khan MN. Palatomaxillary Reconstruction: Fibula or Scapula. Semin Plast Surg 2020;34:86-91.

9. Okay DJ, Genden E, Buchbinder D, et al. Prosthodontic guidelines for surgical reconstruction of the maxilla: a classification system of defects. J Prosthet Dent 2001;86:352-63.

10. Yamamoto Y, Kawashima K, Sugihara T, et al. Surgical management of maxillectomy defects based on the concept of buttress reconstruction. Head Neck 2004;26:247-56.

11. Bender-Heine A, Wax MK. Reconstruction of the Midface and Palate. Semin Plast Surg 2020;34:77-85.

12. Costa H, Zenha H, Sequeira H, et al. Microsurgical reconstruction of the maxilla: Algorithm and concepts. J Plast Reconstr Aesthet Surg 2015;68:e89-e104.

13. Futran ND, Wadsworth JT, Villaret D, et al. Midface reconstruction with the fibula free flap. Arch Otolaryngol Head Neck Surg 2002;128:161-6.

14. Davison SP, Sherris DA, Meland NB. An algorithm for maxillectomy defect reconstruction. Laryngoscope 1998;108:215-9.

15. Frederick JW, Sweeny L, Carroll WR, et al. Outcomes in head and neck reconstruction by surgical site and donor site. Laryngoscope 2013;123:1612-7.

16. Wilkman T, Apajalahti S, Wilkman E, et al. A Comparison of Bone Resorption Over Time: An Analysis of the Free Scapular, Iliac Crest, and Fibular Microvascular Flaps in Mandibular Reconstruction. J Oral Maxillofac Surg 2017;75:616-21.

17. Largo RD, Garvey PB. Updates in Head and Neck Reconstruction. Plast Reconstr Surg 2018;141:271e-85e.

18. Hanasono MM, Silva AK, Yu P, et al. A comprehensive algorithm for oncologic maxillary reconstruction. Plast Reconstr Surg 2013;131:47-60.

19. Vincent A, Burkes J, Williams F, et al. Free Flap Reconstruction of the Maxilla. Semin Plast Surg 2019;33:30-7.

20. Cao Y, Yu C, Liu W, et al. Obturators versus flaps after maxillary oncological ablation: A systematic review and best evidence synthesis. Oral Oncol 2018;82:152-61. 
21. Vander Poorten V, Meulemans J, Delaere P. Midface prosthetic rehabilitation. Curr Opin Otolaryngol Head Neck Surg 2016;24:98-109.

22. Futran ND, Mendez E. Developments in reconstruction of midface and maxilla. Lancet Oncol 2006;7:249-58.

23. Chang JW, Lim JH, Lee JH. Reconstruction of midface defects using local flaps: An algorithm for appropriate flap choice. Medicine (Baltimore) 2019;98:e18021.

24. Li J, Shen Y, Wang L, et al. Superficial Temporal Versus Cervical Recipient Vessels in Maxillary and Midface Free Vascularized Tissue Reconstruction: Our 14-Year Experience. J Oral Maxillofac Surg 2018;76:1786-93.

25. Shaw RJ, Ho MW, Brown JS. Thoracodorsal artery perforator - scapular flap in oromandibular reconstruction with associated large facial skin defects. Br J Oral Maxillofac Surg 2015;53:569-71.

doi: 10.21037/fomm-2021-10

Cite this article as: Lago-Beack B, Acero-Sanz J. Reconstruction of midfacial defects. Front Oral Maxillofac Med 2021;3:27.
26. Rodby KA, Turin S, Jacobs RJ, et al. Advances in oncologic head and neck reconstruction: systematic review and future considerations of virtual surgical planning and computer aided design/computer aided modeling. J Plast Reconstr Aesthet Surg 2014;67:1171-85.

27. Shenaq DS, Matros E. Virtual planning and navigational technology in reconstructive surgery. J Surg Oncol 2018;118:845-52.

28. Wilde F, Schramm A. Intraoperative imaging in orbital and midface reconstruction. Facial Plast Surg 2014;30:545-53.

29. Zhu B, Han M, Heaton C, et al. Assessing Free Flap Reconstruction Accuracy of the Midface and Orbit Using Computer-Aided Modeling Software. Facial Plast Surg Aesthet Med 2020;22:93-9. 